\title{
Características de pacientes superobesos atendidos em serviço de referência em cirurgia bariátrica
}

\author{
Characteristics of overweight patients treated at a referral service in bariatric surgery
}

\section{DOI: $10.37111 /$ braspenj.2019344012}

Francisca Luzia Soares Macieira de Araújo

Edith Monielyck Mendonça Batista ${ }^{2}$

Gutemberg Fernandes de Araújo ${ }^{3}$

Silvana Maria Mendes Costa ${ }^{4}$

Andréa Karine de Araújo Santiago 5

Ana Cláudia Ribeiro Gonçalves

\begin{abstract}
Unitermos:
Obesidade mórbida. Cirurgia bariátrica. Transtorno da compulsão alimentar. Transtorno depressivo. Comorbidade.
\end{abstract}

\section{Keywords:}

Obesity, morbid. Bariatric surgery. Binge-eating disorder. Depressive disorder. Comorbidity.

\section{Endereço para correspondência:}

Francisca Luzia Soares Macieira de Araújo

Rua Miragem do Sol, 27 - apto 601 - Residencial Broadway - Renascença II - São Luís, MA, Brasil CEP 65075-760

E-mail francisca.lsm@gmail.com

\section{Submissão}

25 de julho de 2019

Aceito para publicação

10 de novembro de 2019

\begin{abstract}
RESUMO
Introdução: A obesidade é uma epidemia mundial e pessoas com superobesidade (IMC $\geq 50 \mathrm{~kg}$ ) $\mathrm{m}^{2}$ ) têm pior prognóstico, estando relacionada com complicações físicas, mas também com doenças mentais, abuso físico e verbal, além de exclusão social. Objetivo: Determinar o perfil de pacientes superobesos atendidos em serviço de referência em cirurgia bariátrica. Método: Realizada análise retrospectiva de 56 prontuários de pacientes atendidos no ambulatório multidisciplinar do Serviço de Cirurgia Bariátrica e Metabólica do HUUFMA, no período de 2017 a 2019. Foram excluídos prontuários incompletos e com índice de massa corpórea $(I M C)<50 \mathrm{~kg} / \mathrm{m}^{2}$. Os resultados estão apresentados em média e desvio padrão. Utilizou-se o teste do qui-quadrado para a análise de associações, e o de Shapiro-Wilk para a normalidade e considerou-se significância $p<0,05$. Resultados: Prevalência na amostra do sexo feminino $(80,3 \%)$, pardas $(67,8 \%)$, idade média de $37,8 \pm 9,8$ anos e $49,1 \%$ conclúram o ensino médio. O peso e o IMC médio foram de 136,6 \pm $12,4 \mathrm{~kg}$ e $59 \pm 6,5 \mathrm{~kg} / \mathrm{m}^{2}$, respectivamente, destacando-se um paciente com IMC de $71 \mathrm{~kg} / \mathrm{m}^{2}$. Dentre as comorbidades encontramos apneia obstrutiva do sono em $100 \%$, insônia em $98,2 \%$, problemas articulares em $53,5 \%$, ansiedade em $50 \%$, esteatose hepática e hipertensão arterial sistêmica com $48,2 \%$ cada, dislipidemia em $42,8 \%, 32,1 \%$ apresentavam constipação intestinal e $17,8 \%$ diabetes melito tipo 2. Houve relação significante $(p<0,05)$ entre escolaridade e peso. Observou-se impacto na saúde mental, sendo que $37,5 \%$ dos pacientes apresentavam depressão, $44,6 \%$ compulsão alimentar e $17,6 \%$ pensamentos suicidas ou de automutilação. Conclusão: 0 perfil dos pacientes superobesos é de mulheres adultas jovens, com escolaridade média, comorbidades variadas e com impacto significativo na saúde mental.
\end{abstract}

\section{ABSTRACT}

Introduction: Obesity is a worldwide epidemic and people with super obesity (BMI > $50 \mathrm{~kg} / \mathrm{m}^{2}$ ) have the worse prognosis, related to physical complications but also to mental illnesses, physical and verbal abuse, as well as social exclusion. Objective: To determine the profile of super obese patients treated in a referential bariatric surgery service. Methods: Retrospective analysis of 56 medical records of patients treated at the HUUFMA's Bariatric and Metabolic Surgery Service multidisciplinary ambulatory care in 2017-2019. Incomplete records or with body mass index (BMI) < $50 \mathrm{~kg} / \mathrm{m}^{2}$ were excluded. Results are presented in mean and standard deviation. Chi-square test was used for association analysis, Shapiro-Wilks test for normality and significance was considered for $p<0.05$. Results: Prevalence in sample of females $(80.3 \%)$, brown skin $(67.8 \%)$, average age of $37.8 \pm 9.8$ years, and $49.1 \%$ graduated high school. The average weight and BMl were that of $136.6 \pm 12.4 \mathrm{~kg}$ and $59 \pm 6.5 \mathrm{~kg} / \mathrm{m}^{2}$, respectively, with a patient standing out with a BMI of 71 $\mathrm{kg} / \mathrm{m}^{2}$. Among the comorbidities we found obstructive sleep apnea in $100 \%$, insomnia in $98.2 \%$, joint problems in $53.5 \%$, anxiety in $50 \%$, hepatic steatosis and systemic arterial hypertension with $48.2 \%$ each, dyslipidemia in $42.8 \%$, intestinal constipation in $32.1 \%$ and type 2 diabetes mellitus in $17.8 \%$. There was a significant relation $(p<0.05)$ between schooling and weight. An impact on mental health was observed, with $37.5 \%$ of the patients exhibiting depression, $44.6 \%$ binge eating and $17.6 \%$ suicidal thoughts and/or self-mutilation. Conclusion: The super obese patients' profile is that of a young adult woman, with average schooling, various comorbidities and with significant impact on mental health.

1. Mestre em Ciências da Saúde pela Universidade Federal do Maranhão (UFMA), Professora Adjunta da UFMA, Curso de Medicina, DEMED I, Médica Clínica, São Luís, MA, Brasil.

2. Aluna do Curso de Medicina da Universidade Federal do Maranhão, São Luís, MA, Brasil.

3. Doutor em Cirurgia Experimental pela Escola Paulista de Medicina, Professor Adjunto da Universidade Federal do Maranhão, Curso de Medicina, DEMED II, Cirurgião Bariátrico Titular da Sociedade Brasileira de Cirurgia Bariátrica e Metabólica, Coordenador do Serviço de Cirurgia Bariátrica e Metabólica do Hospital Universitário da UFMA (HUUFMA), São Luís, MA, Brasil.

4. Enfermeira do Serviço de Cirurgia Bariátrica e Metabólica do Hospital Universitário da UFMA (HUUFMA), São Luís, MA, Brasil.

5. Especialista em Nutrição e Alimentos Universidade Federal do Piauí, Nutricionista do Serviço de Cirurgia Bariátrica e Metabólica do Hospital Universitário da UFMA (HUUFMA), São Luís, MA, Brasil.

6. Especialista em Intervenções Nutricionais UFMA, Especialista em Nutrição Clínica Universidade Gama Filho, Especialista em Nutrição Entera e Parenteral pelo GANEP, Nutricionista do Serviço de Cirurgia Bariátrica e Metabólica do Hospital Universitário da UFMA (HUUFMA), São Luís, MA, Brasil. 


\section{INTRODUÇÃO}

A obesidade é uma epidemia mundial e deve ser vista como um grave problema de saúde pública, ${ }^{1,2}$. Contribui para 0 aparecimento de comorbidades, como diabetes mellitus, doenças cardiovasculares, hipertensão arterial sistêmica (HAS), apneia obstrutiva do sono, doenças musculoesqueléticas, acidentes vasculares cerebrais e piora da qualidade de vida ${ }^{1-3}$. O risco de morte aumenta com o grau de obesidade, sendo mais exacerbado para índice de massa corporal (IMC) $\geq 35 \mathrm{~kg} / \mathrm{m}^{2}$, causando efeitos não somente à saúde ${ }^{4,5}$, mas também à economia, aumentando a utilização e os gastos com serviços assistenciais ${ }^{6}$. A população com superobesidade (IMC $\geq 50 \mathrm{~kg} / \mathrm{m}^{2}$ ) aumentou $500 \%$ na última década, representando $30 \%$ a $50 \%$ dos obesos graves ${ }^{7}$. Pessoas com superobesidade têm pior prognóstico, estando relacionado com complicações físicas, mas também a doenças mentais, abuso físico e verbal, além de exclusão social. Como resultado, ocorre aumento da demanda pelo tratamento cirúrgico para obter perda ponderal ${ }^{1,7}$, sendo pacientes que apresentam maiores dificuldades técnicas e maior incidência de comorbidades clínicas e psiquiátricas ${ }^{2,6,7}$. Entre estas alterações destaca-se o transtorno da compulsão alimentar periódica (TCAP), pois parece haver correlação positiva entre a gravidade do TCAP e o grau de obesidade ${ }^{6}$.

As informações sobre indivíduos superobesos são escassas na literatura internacional e nacional ${ }^{6}$. É necessário conhecer sua prevalência e os fatores associados que os distinguem dos demais obesos, os aspectos sociodemográficos, comorbidades e os reflexos na saúde mental desta população $0^{6,7}$.

O objetivo deste estudo é determinar o perfil de pacientes superobesos atendidos em senviço de referência em cirurgia bariátrica.

\section{MÉTODO}

Estudo transversal com análise retrospectiva de 56 prontuários de pacientes portadores de superobesidade (IMC $\left.\geq 50 \mathrm{~kg} / \mathrm{m}^{2}\right)$ atendidos no ambulatório multidisciplinar do Serviço de Cirurgia Bariátrica e Metabólica do Hospital Universitário da Universidade Federal do Maranhão (HUUFMA), São Luís-Maranhão, de 2017 a 2019. Foram excluídos pacientes com prontuários incompletos e com $I M C<50 \mathrm{~kg} / \mathrm{m}^{2}$. As informações foram coletadas das evoluções dos prontuários eletrônicos e impressos, utilizando-se uma planilha Microsoft Excel para arquivamento das variáveis: dados sociodemográficos (idade, cor da pele, anos de estudo), presença de comorbidades (HAS e doenças cardíacas, diabetes mellitus, dislipidemias, doenças gastrintestinais, doenças osteoarticulares), TCAP, alterações psicológicas (depressão, pensamentos suicidas e automutilação). A TCAP foi avaliada pela Escala de Compulsão Alimentar Periódica (ECAP) traduzida e adaptada por Freitas et al. ${ }^{8}$. O cálculo do IMC foi feito após coleta do peso e altura, utilizando-se balança tipo plataforma Welmy com capacidade até $300 \mathrm{~kg}$, escala de $100 \mathrm{~g}$, com os participantes vestindo roupas leves e descalços para aferição do peso e a altura foi medida utilizando antropômetro vertical milimetrado da balança tipo plataforma, com escala de $0,1 \mathrm{~cm}$. Os dados de peso e altura foram coletados segundo técnica proposta pela Organização Mundial da Saúde ${ }^{7}$. A pressão arterial foi aferida por esfigmomanômetro marca Premium, com manguito de 31 a $51 \mathrm{~cm}$, adaptado para obeso. As comorbidades foram definidas conforme o Quadro 1.

Quadro 1 - Definição das comorbidades.

\begin{tabular}{ll}
\hline Comorbidade & \multicolumn{1}{c}{ Definição } \\
\hline Hipertensão arterial sistêmica & PAS $\geq 140 \mathrm{mmHg}$ e PAD $\geq 90 \mathrm{mmHg}$ \\
& $7^{\text {a }}$ Diretriz Brasileira de Hipertensão Arterial ${ }^{9}$ \\
\hline Diabete mellitus & Glicemia de jejum $\geq 126 \mathrm{mg} / \mathrm{dL}$ ou casual $\geq 200 \mathrm{mg} / \mathrm{dL}$ ou A1c $\geq 6,5 \%$ \\
& American Diabetes Association $2013^{10}$ \\
\hline Dislipidemia & Colesterol total $<200 \mathrm{mg} / \mathrm{dL}, \mathrm{LDL}-\mathrm{c}<160 \mathrm{mg} / \mathrm{dL}, \mathrm{HDL}-\mathrm{C}>50 \mathrm{mg} / \mathrm{dL}$ para mulheres e $>40 \mathrm{mg} / \mathrm{dL}$ para \\
& homens e triglicérides $<150 \mathrm{mg} / \mathrm{dL}$ \\
& Atualização da Diretriz Brasileira de Dislipidemias e Prevenção da Aterosclerose - 2017 ${ }^{11}$ \\
\hline Transtorno da compulsão alimentar & Diagnostic and Statistical Manual of Mental Disorders (DSM-5). American Psychiatry Association ${ }^{12}$ \\
\hline periódica & No mínimo uma queixa: 1. Episódios de sono não intencionais durante a vigília, sonolência excessiva \\
\hline Apneia obstrutiva do sono & diurna, sono não reparador, fadiga ou insônia; 2. Acordar com pausas respiratórias, engasgos ou asfixia; \\
& 3. Companheiro relatar ronco alto e/ou pausas respiratórias no sono ${ }^{13}$ \\
& Critérios diagnósticos e tratamento dos distúrbios respiratórios do sono: SAOS ${ }^{13}$ \\
\hline Doenças osteoarticulares & Exame clínico + imagem (radiografia, tomografia computadorizada ou ressonância magnética) e avalia- \\
\hline ção do especialista \\
\hline Ansiedade & Diagnostic and Statistical Manual of Mental Disorders (DSM-5). American Psychiatry Association ${ }^{12}$ \\
\hline
\end{tabular}

$\overline{\mathrm{PAS}}$ = pressão arterial sistólica; $\mathrm{PAD}$ = pressão arterial diastólica; $S A O S=$ síndrome de apneia obstrutiva do sono. 
Para análise estatística, os resultados foram apresentados em média e desvio padrão. Utilizou-se o teste do qui-quadrado para a análise de associações, Shapiro-Wilk para a normalidade e considerou-se significância $p<0,05$. A análise estatística foi realizada no Stata 8.0.

\section{RESULTADOS}

Na amostra estudada, encontramos prevalência do sexo feminino $(n=45,80,3 \%)$, cor parda $(n=38,67,8 \%)$, idade média de 37,8 $\pm 9,8$ anos e 49,1\% concluíram o ensino médio, com tempo médio de estudo de $16,5 \pm 2,3$ anos. O peso e o IMC médio foram 136,6 $\pm 12,4 \mathrm{~kg}$ e $59 \pm 6,5$ $\mathrm{kg} / \mathrm{m}^{2}$, respectivamente, destacando-se um paciente com IMC de $71 \mathrm{~kg} / \mathrm{m}^{2}$ (Tabela 1).
Dentre as comorbidades, encontramos apneia obstrutiva do sono clinicamente identificada em $100 \%$ dos pacientes, insônia em $55(98,2 \%)$, problemas articulares em 30 (53,5\%), ansiedade em $50 \%$, esteatose hepática e HAS em $27(48,2 \%)$ cada, dislipidemia identificada em 24 pacientes $(42,8 \%), 18$ $(32,1 \%)$ apresentavam constipação intestinal e $10(17,8 \%)$ eram portadores de diabetes mellitus 2 (Figura 1).

Houve relação significante entre escolaridade e peso $(p<0,05)$.

Observou-se impacto na saúde mental, sendo que 44,6\% apresentaram TCAP, 37,5\% transtorno depressivo e 17,6\% pensamentos suicidas ou de automutilação (Figura 2).

As características sociodemográficas dos pacientes avaliados encontram-se resumidas na Tabela 2 .

Tabela 1 - Distribuição dos dados antropométricos.

\begin{tabular}{lccccc}
\hline Variável & Média & DP & Mínimo & Mediana & Máximo \\
\hline Peso $(\mathrm{kg})$ & 136,6 & 12,4 & 114 & 144,5 & 217 \\
$\mathrm{IMC}\left(\mathrm{kg} / \mathrm{m}^{2}\right)$ & 59 & 6,5 & 50,1 & 53,6 & 70,9 \\
\hline
\end{tabular}

$\mathrm{DP}$ = desvio padrão; $\mathrm{IMC}$ = índice de massa corporal.

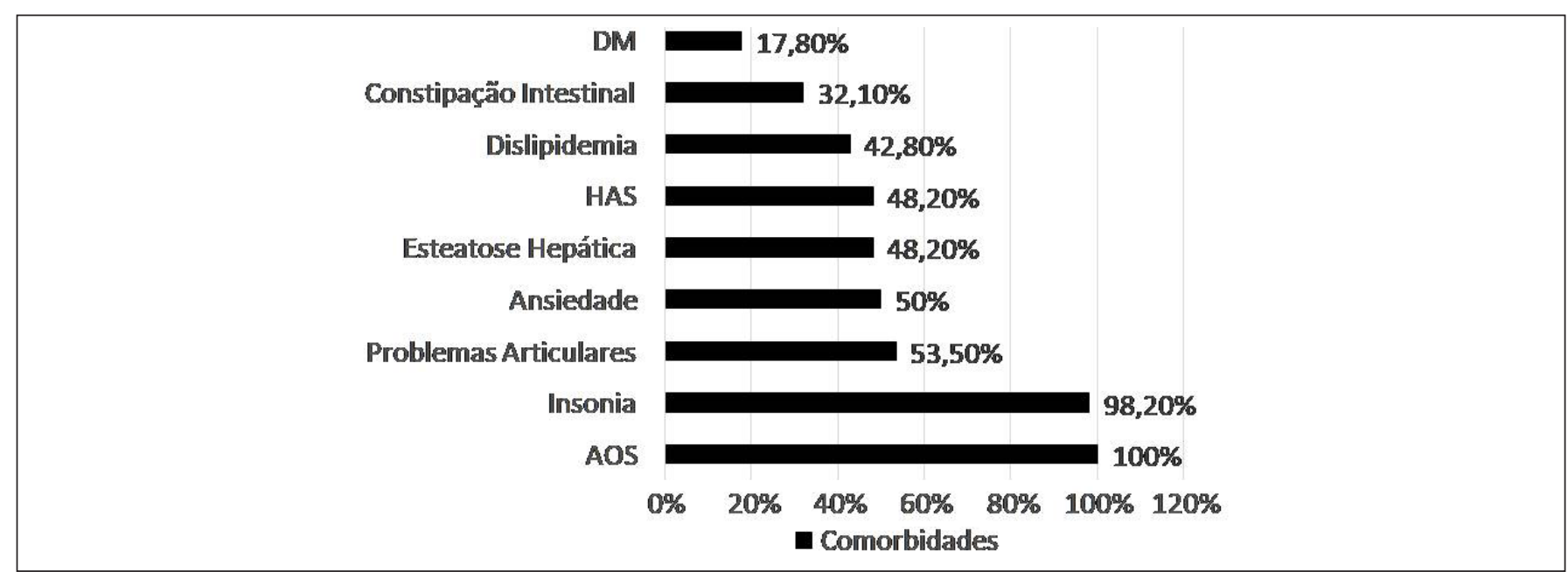

Figura 1 - Percentual de comorbidades. AOS = apneia obstrutiva do sono; DM = diabetes mellitus; HAS = hipertensão arterial sistêmica.

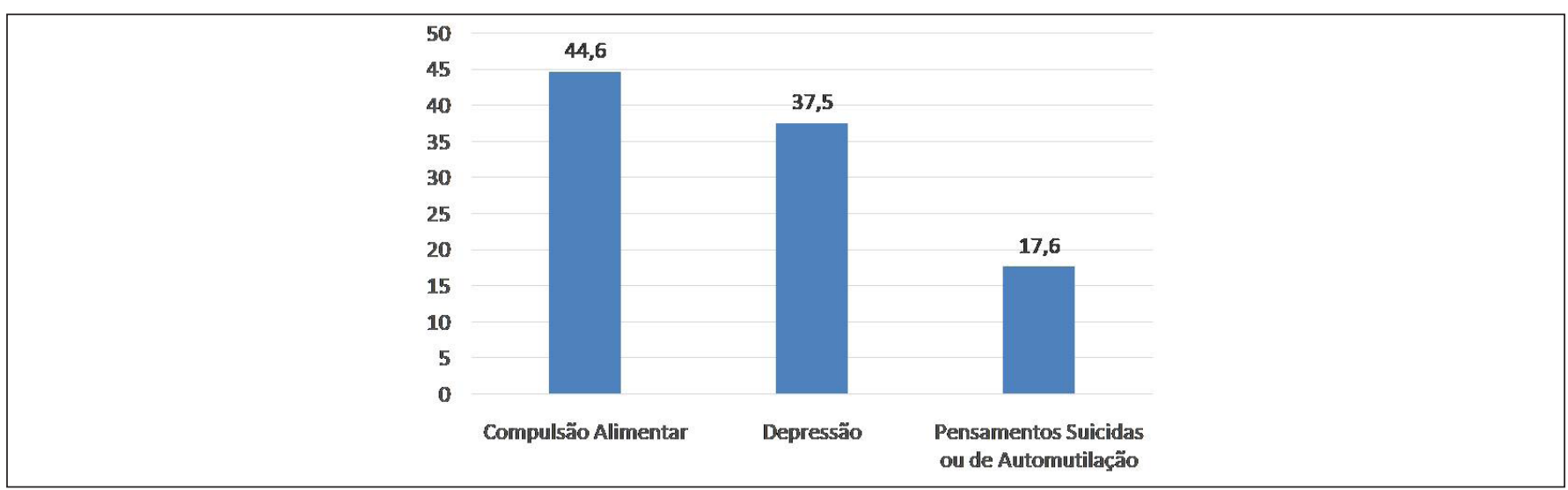


Tabela 2 - Características sociodemográficas dos pacientes.

\begin{tabular}{lc}
\hline Característica & $\mathbf{N}$ \\
\hline Sexo & $45(80,3 \%)$ \\
Feminino & $11(19,7 \%)$ \\
Masculino & \\
\hline Raça & $38(67,8 \%)$ \\
Pardo & $10(17,9 \%)$ \\
Branco & $8(14,3 \%)$ \\
Negro & $37,8 \pm 9,8$ anos \\
\hline Idade & \\
\hline Estado Civil & $11(19,7 \%)$ \\
Solteiro & $18(32,2 \%)$ \\
Casado & $24(42,8 \%)$ \\
União estável & $2(3,6 \%)$ \\
Divorciado & $1(1,7 \%)$ \\
Viúvo & $16,5 \pm 2,3$ anos \\
\hline Escolaridade em anos de estudos & $12(21,3 \%)$ \\
\hline Ocupação & $8(14,3 \%)$ \\
Desempregado & $18(32,2 \%)$ \\
Estudante & $18(32,2 \%)$ \\
Dona de casa &
\end{tabular}

\section{DISCUSSÃO}

O perfil clínico dos participantes do estudo foi predominantemente constituído por mulheres $(80,3 \%)$, adultas e com comorbidades associadas, similar aos dados de outros estudos brasileiros. Camargo et al. ${ }^{14}$ destacam a prevalência de pacientes do sexo feminino (83,8\%), adultas, com obesidade grau III, atribuindo este fato à procura maior das mulheres pelo procedimento cirúrgico e também que o realizam em busca de melhora das comorbidades e da qualidade de vida. Dado semelhante foi encontrado nos trabalhos de Ferreira et al. ${ }^{15}$, com $80 \%$ de sexo feminino, e Steyer et al. ${ }^{16}$, com prevalência de $84 \%$ de sexo feminino nas amostras estudadas.

A idade média dos pacientes deste estudo foi de 37,8 $\pm 9,8$ anos, semelhante ao perfil das obesas descritas por Rodrigues e Silveira ${ }^{6}$, com faixa etária de 40,9 $\pm 12,1$ anos e Steyer et al. ${ }^{16}$, com idade média de $39 \pm 10,1$ anos, mais velhas que as estudadas por Ferreira et al. ${ }^{15}$, que tinham idade média de 33 anos.

A relação significante entre escolaridade e peso encontrada nesta amostra é também relatada por Rodrigues e Silveira ${ }^{17}$, que reportaram que as mulheres com 0 a 8 anos de estudo tinham risco 2,38 vezes maior de serem superobesas em relação às mulheres com 9 anos de estudo ou mais.

Nesta pesquisa, os pacientes apresentaram peso médio de $136,6 \mathrm{~kg}$ e IMC médio de $59,0 \mathrm{~kg} / \mathrm{m}^{2}$. Ferreira et al. ${ }^{15}$ relataram peso médio de 130,25 kg e IMC médio de 49,04 $\mathrm{kg} / \mathrm{m}^{2}$, mais baixos que desta amostra. Rodrigues e Silveira ${ }^{6}$, estudando 72 mulheres, encontraram prevalência de superobesidade em $36,1 \%$ e IMC de 55,8 $\pm 5,3 \mathrm{~kg} / \mathrm{m}^{2}$ entre as superobesas.

Em relação às comorbidades, a apneia obstrutiva do sono identificada clinicamente foi que apresentou maior prevalência, seguida de insônia, corroborando o dado anterior ${ }^{18}$. Essa elevada incidência pode ser justificada pelo maior peso dos pacientes em relação a outros estudos divergentes na prevalência da principal comorbidade, como o de Ferreira et al. ${ }^{15}$, que encontraram predominância de HAS em $60 \%$ dos pacientes, semelhante ao trabalho de Rodrigues e Silveira 6 , com $58,3 \%$ de HAS e Steyer et al. ${ }^{16}$, com $72 \%$ de hipertensos no estudo.

Os problemas articulares foram diagnosticados em 53,5\% dos pacientes, superior à incidência encontrada por Steyer et al. ${ }^{16}$, de $30 \%$, refletindo a ação continuada do peso elevado sobre as articulações.

O TCAP foi identificado em $44,6 \%$ dos pacientes estudados na presente pesquisa. No estudo de Rodrigues e Silveira ${ }^{6}$, sobre fatores associados à superobesidade em mulheres, 51,6\% dos pacientes apresentaram comportamento de compulsão alimentar, sendo que as superobesas tinham risco 2,41 vezes maior de ter compulsão alimentar. Melo et al. ${ }^{19}$ identificaram a presença de compulsão alimentar em $60 \%$ de superobesos (IMC 50 a 59,9 kg/m²) e 70\% em super superobesos (IMC $\left.\geq 60 \mathrm{~kg} / \mathrm{m}^{2}\right)$.

Pacientes superobesos, frequentemente, experimentam estresse aumentado pela discriminação relacionada à obesidade e os pacientes bariátricos relatam isolamento social como resultado do excesso de peso', o receio de dizer ou fazer alguma coisa "errada", de ser julgado negativamente pela aparência e, em aproximadamente $36 \%$ dos indivíduos obesos, associa-se com TCAP', criando um círculo vicioso que leva ao transtorno depressivo, presente em $37,5 \%$ dos pacientes desta amostra. Silva e Lange ${ }^{20}$, em revisão sobre a imagem corporal feminina, verificaram que a obesidade em indivíduos do sexo feminino desencadeia aspectos psicológicos de baixa autoestima, ansiedade, angústia, agressividade, tristeza, compulsão, negação e insatisfação com a imagem corporal. Esses sentimentos negativos consigo mesmos explicaria, de certa forma, as tentativas de suicídio e as automutilações referidos por $17,6 \%$ dos pacientes.

\section{CONCLUSÃO}

O perfil dos pacientes superobesos neste estudo é de mulheres adultas jovens, com escolaridade média, comorbidades variadas e impacto significativo na saúde mental. 


\section{REFERÊNCIAS}

1. Fencl JL, Walsh A, Vocke D. The bariatric patient: an overview of perioperative care. AORN J. 2015;102(2):116-31.

2. Wannmacher L. Obesidade como fator de risco para morbidade e mortalidade: evidências sobre o manejo com medidas não medicamentosas. PAHO. 2016;1(7):1-10.

3. Di Cesare M, Bentham J, Stevens GA, Zhou B, Danaei G, Lu $\mathrm{Y}$, et al. Trends in adult body-mass index in 200 countries from 1975 to 2014: a pooled analysis of 1698 population-based measurement studies with 19.2 million participants. Lancet. 2016;387(10026):1377-96.

4. Kitahara CM, Flint AJ, Berrington de Gonzalez A, Bernstein L, Brotzman M, MacInnis RJ, et al. Association between class III obesity (BMI of 40-59 $\mathrm{kg} / \mathrm{m}^{2}$ ) and mortality: a pooled analysis of 20 prospective studies. PLoS Med. 2014;11(7):e1001673.

5. The Global BMI Mortality Collaboration. Di Angelantonio E, Bhupathiraju ShN, Wormser D, Gao P, Kaptoge S, Berrington de Gonzalez A, et al. Body-mass index and all-cause mortality: individual-participant-data meta-analysis of 239 prospective studies in four continents. Lancet. 2016;388(10046):776-86.

6. Rodrigues APS, Silveira EA. Fatores associados à superobesidade em mulheres: compulsão alimentar periódica e consumo alimentar. RBONE. 2018;12(73):643-54.

7. Santo MA, Riccioppo D, Pajecki D, Cleva R, Kawamoto F, Cecconello I. Preoperative weight loss in super-obese patients: study of the rate of weight loss and its effects on surgical morbidity. Clinics. 2014;69(12):828-34.

8. Freitas S, Lopes CS, Coutinho W, Appolinario JC. Tradução e adaptação para o português da Escala de Compulsão Alimentar Periódica. Rev Bras Psiquiatr. 2001;23(4):215-20.

9. Sociedade Brasileira de Cardiologia. Malachias MVB, Souza WKSB, Plavnik FL, Rodrigues CIS, Brandão AA, Neves MFT, et al. $7^{\text {a }}$ diretriz brasileira de hipertensão arterial. Arq Bras Cardiol. 2016;107(3 Supl.3):1-83.
10. American Diabetes Association. Diagnosis and classification of diabetes mellitus. Diabetes Care. 2010;33(Suppl. 1):S62-9.

11. Faludi AA, Izar MCO, Saraiva JFK, Chacra APM, Bianco HT, Afiune Neto A, et al. Atualização da Diretriz Brasileira de Dislipidemias e Prevenção da Aterosclerose-2017. Arq Bras Cardiol. 2017;109(2 Supl.1):1-76.

12. American Psychiatry Association. Diagnostic and statistical manual of mental disorders (DSM-5). $5^{\text {th }}$ ed. Washington: American Psychiatry Association; 2013.

13. BittencourtLRA, CaixetaEC. Critérios diagnósticos e tratamento dos distúrbios respiratórios do sono: SAOS. J Bras Pneumol. 2010;36(suppl. 2):23-7.

14. Camargo SMPLO, Masari SP, Inácio TAF. Preparação multidisciplinar pré-cirurgia bariátrica na visão do cliente. RBONE. 2012;6(32):123-8.

15. Ferreira JDC, Pedroso CF, Sousa TR. Perfil clínico e avaliação da dor de pacientes obesos no período pré-operatório de cirurgia bariátrica. Rev Cien Escol Estad Saud Publ Cândido SantiagoRESAP. 2017;3(2):82-96.

16. Steyer NH, Oliveira MC, Gouvêa MRF, Echer IC, Lucena AF. Perfil clínico, diagnósticos e cuidados de enfermagem para pacientes em pós-operatório de cirurgia bariátrica. Rev Gaúcha Enferm. 2016;37(1):e5017.

17. Rodrigues APS, Silveira EA. Correlação e associação de renda e escolaridade com condições de saúde e nutrição em obesos graves. Ciênc Saúde Coletiva. 2015;20(1):165-74.

18. Silva GA, Sander HH, Eckeli AL, Fernandes RMF, Coelho EB, Nobre F. Conceitos básicos sobre síndrome da apneia obstrutiva do sono. Rev Bras Hipertens. 2009;16(3):150-7.

19. Melo PG, Peixoto MRG, Silveira EA. Binge eating prevalence according to obesity degrees and associated factors in women. J Bras Psiquiatr. 2015;64(2):100-6.

20. Silva GA, Lange ESN. Imagem corporal: a percepção do conceito em indivíduos obesos do sexo feminino. Psicol Argum. 2010;28(60):43-54.

\footnotetext{
Local de realização do estudo: Hospital Universitário da Universidade Federal do Maranhão (HUUFMA), Serviço de Cirurgia Bariátrica e Metabólica, São Luís, MA, Brasil.
}

Conflito de interesse: Os autores declaram não haver.

Trabalho apresentado como pôster destaque no XXIII Congresso Brasileiro de Nutrição Parenteral e Enteral, Foz do Iguaçu, 21 de outubro de 2019. 